\title{
Создание равномерных покрытий импульсным лазерным осаждением
}

\author{
Н. И. Анякин ${ }^{\mathrm{a}^{* * * *}}$, Р. О. Жук ${ }^{\mathrm{a}^{* * *}}$, Е. В. Проценко ${ }^{\mathrm{a}}$, \\ В. С. Коваленко ${ }^{a}$, А. Н. Степура ${ }^{a}$, Д. Яо \\ ${ }^{a}$ Национальный технический университет Украинь имени Игоря Сикорского \\ «Киевский политехнический институт», Киев-56, 03056, Украина, \\ ${ }^{b}$ Исследовательский иентр лазерной обработки и техники, Чжэизянский университет технологий, КНР

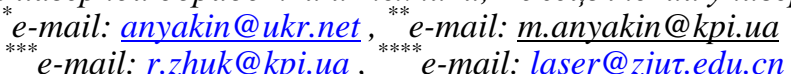 \\ Поступила в редакцию 11.02.2021 \\ После доработки 26.04.2021 \\ Принята к публикации 30.04.2021
}

Рассмотрен выбор режимов лазерной обработки, которые позволяют получать тонкие слои напыленного материала равномерной толщины методом импульсного лазерного осаждения. Исследования проводились в атмосфере Ar, в качестве материала для напыления использовали образцы из кремния и карбида кремния. Обработка результатов предварительных экспериментов позволила установить связь между яркостью изображения напыленного слоя (при условии его равномерного освещения) и его толщиной. Установлена зависимость толщины напыленного слоя от пространственных координат для различных режимов обработки и с помощью расчетов и выбрана закономерность перемещения заготовки, которая обеспечивает получение напыленных слоев с минимальным отклонением от заданной толщины. На основе результатов технология лазерного осаждения использовалась для создания покрытия на заготовке большой площади.

Ключевые слова: лазер, лазерная технология, импульсное лазерное осаждение, оптимизация

УДК 621.373.8

https://doi.org/10.52577/eom.2021.57.6.25

\section{ВВЕДЕНИЕ}

Использование технологии импульсного лазерного осаждения (ИЛО) позволяет создавать на поверхности заготовки сверхтонкие пленки из любых материалов [1]. Причем высота напыленного слоя $H$ зависит от:

- режимов лазерной обработки (энергии лазерного излучения $E_{p}$, длительности импульса $\tau_{p}$, фокусного расстояния, фокусирующего объектива $F$, расходимости излучения $\Theta$, размера пятна фокусирования $d_{p}$, длины волны излучения $\lambda$ и др.);

- теплофизических, оптических свойств мишени и заготовки, расстояния между ними $S$;

- свойств окружающей среды (вакуум, рабочий газ, его давление и пр.) и др., и изменяется от нанометра до нескольких микрометров [1]. Очевидно, что вблизи оси плазменного факела, который возникает при испарении поверхности мишени и «оседает» на поверхности заготовки, высота $H$ и «плотность» напыленного слоя наибольшие. Естественно, при создании напыленного покрытия равномерной высоты большой площади [2-4] прежде всего необходимо установить зависимость высоты напыленного слоя (который образуется на поверхности заготовки за счет испарения поверхности мишени под действием отдельного лазерного импульса) от пространственных координат $H(x, y)$ для различных режимов обработки. В последующем необходимо определить взаимное расположение напыленных пятен на поверхности заготовки, которое обеспечивает заданные свойства покрытия (высоту и допустимое ее колебание). То есть, например, для данных режимов обработки определяются скорость и закон перемещения заготовки.

Данная работа посвящена разработке простого метода определения $H(x, y)$ на поверхности заготовки, полученного с использованием ИЛО, созданию на ее поверхности равномерных покрытий заданной высоты $H$ (с минимальным еe колебанием), в том числе покрытий на подложки большой площади.

\section{МЕТОДИКА ПРОВЕДЕНИЯ \\ ЭКСПЕРИМЕНТОВ И ИСПОЛЬЗУЕМОЕ ОБОРУДОВАНИЕ}

При проведении исследований использовали АИГ-лазер ( $\lambda=1,06$ мкм), который работает в импульсно-периодическом режиме (энергия импульса $E_{p}=1-1,5$ Дж, длительность импульса $\tau_{p}=200$ мкс) с частотой следования импульсов 1-20 Гц. После прохождения двукратной телескопической системы лазерное излучение (рис. 1) фокусировалось объективом с фокусным расстоянием $\mathrm{F}=70$ мм на поверхности мишени, которая устанавливалась под углом $\alpha$ (менялся от $90^{\circ}$ до $40^{\circ}$ ) к оси сфокусированного лазерного излучения. В качестве мишени применяли пластины моно- и поликристаллического кремния Si и карбида кремния SiC. Выбор Si был 


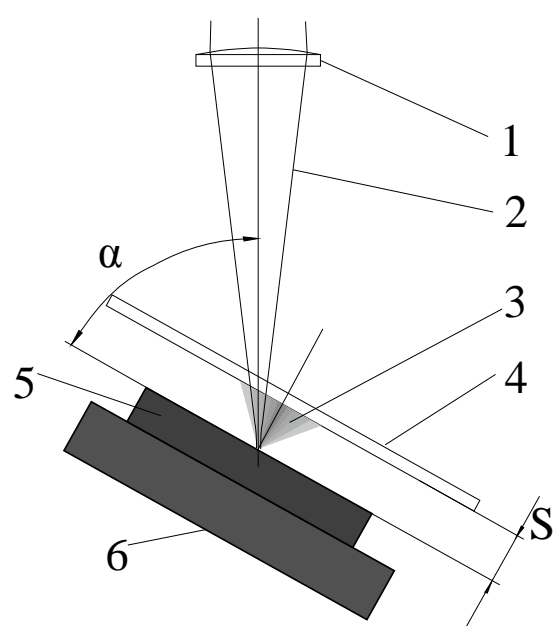

Рис. 1. Схема проведения экспериментов, где 1 - фокусирующая линза; 2 - каустическая поверхность; 3 - плазменное облако; 4 - стеклянная пластина (заготовка); 5 - мишень; 6 - базовая поверхность; $\alpha$ - угол между поверхностью мишени и осью лазерного излучения.

связан с тем, что технологию ИЛО возможно использовать для создания сверхтонких солнечных элементов путем напыления материала мишени на подложку, изготовленную из элементов третьей группы Периодической системы Д.И. Менделеева. Поверхность мишени устанавливалась в фокальной плоскости фокусирующего объектива. Параллельно поверхности мишени на расстоянии $S$, которое менялось от 1 до 5 мм, располагалась стеклянная пластина (стекло К-8 толщиной 1,5 мм), на которую оседали продукты лазерной эрозии. Выбор стекла К-8 связан с идеальным качеством поверхности (шероховатость поверхности не более 0,2 мкм) и «прозрачностью» ее для лазерного излучения. Мишень и стеклянная пластина имели возможность перемещаться параллельно поверхности базирования, причем независимо друг от друга. В отличие от стандартной технологии ИЛО (осуществляется в вакууме или среде рабочего газа под различным давлением [1, 4-6]), эксперименты проводились на воздухе с подачей в зону обработки защитного газа аргона Ar. В каждой экспериментальной точке опыты повторяли три раза. В дальнейшем образцы подлежали УЗ-очистке в воде в течение 10 мин и просушивались сжатым воздухом. Фотографирование поверхности образца после обработки осуществлялось с помощью фотокамеры DCM130 (разрешение 1,3 Мп), установленной в микроскоп МБС-10 в отраженном и в проходящем свете. Освещение образцов происходило с помощью LED фонарей. Высоту напыленного слоя определяли с помощью микроскопа МИС-11 и интерферометра МИИ-4. Профиль напыленного слоя фиксировался фотокамерой с шагом 0,5 и 0,3 мм. В последующем полученные изображения (после определения увеличения системы и обрезания лишних пикселей) объединяли и измеряли высоту профиля. Во избежание системных погрешностей при перемещении предметного столика микроскопа измерение проводили три раза с постоянным перемещением заготовки. Проверку полученных результатов (на установленных режимах обработки) осуществляли при напылении $\mathrm{Si}$ на алюминиевую $\mathrm{Al}$ пластину толщиной 1 мм с последующим изготовлением шлифов и измерением толщины напыленного слоя с помощью растрового электронного микроскопа РЭМ-106И.

\section{ПОЛУЧЕННЫЕ РЕЗУЛЬТАТЫ И ИХ ОБСУЖДЕНИЕ}

Как показали предварительные расчеты, при фокусировании лазерного излучения с $E_{p}>1$ Дж, $\tau_{p}=200$ мкс в пятно $d_{p}=100$ мкм (определялась как $d_{p}=\mathrm{F} \Theta$ ) процесс испарения мишени и образования плазменного облака начинается во время действия лазерного импульса (рис. 2), что сказывается на результате ИЛО. Так, на рис. 2 приведены зависимости температуры поверхности $T(0, t)$ образцов (из различных материалов) и плотности мощности лазерного излучения $W_{p}(t)\left(E_{p}=1,5\right.$ Дж, $\tau_{p}=200$ мкс, $d_{p}=100$ мкм $)$ от времени $t$, полученные при решении многомерного нелинейного нестационарного уравнения теплопроводности с учетом фазовых превращений.

Как видно из приведенных зависимостей, начиная со времени 10-20 мкс (от начала действия импульса), сфокусированное лазерное излучение начнет взаимодействовать с плазменным облаком. Так, при фокусировке лазерного излучения на поверхность мишени (перпендикулярной оси сфокусированного лазерного луча, то есть $\alpha=90^{\circ}$ ) и расположении стеклянной пластины над ее поверхностью на 


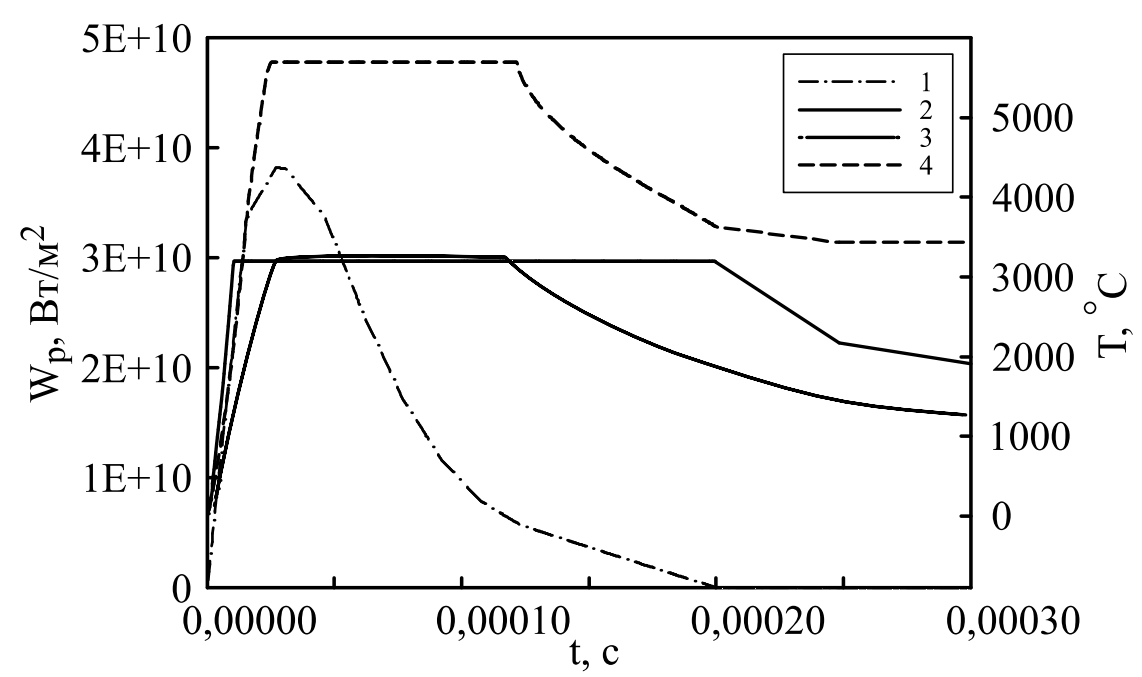

Рис. 2. Зависимости плотности мощности лазерного излучения $W_{p}(t)\left(E_{p}=1,5\right.$ Дж, $\tau_{p}=200$ мкс, $d_{p}=100$ мкм$)$, которая подводится к образцам (из различных материалов), и зависимости температуры $T(0, t)$ их поверхности от времени $t$, где: 1 - зависимость $W_{p}(t) ; 2$ - зависимость температуры поверхности $T(0, t)$ образца из железа; 3 - зависимость температуры поверхности $T(0, t)$ образца из кремния; 4 - зависимость температуры поверхности $T(0, t)$ образца из вольфрама.

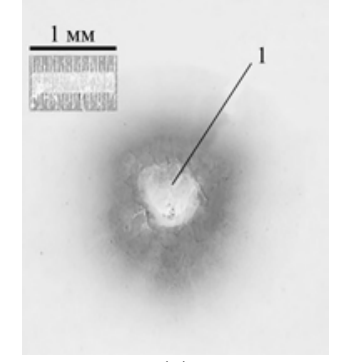

(a)

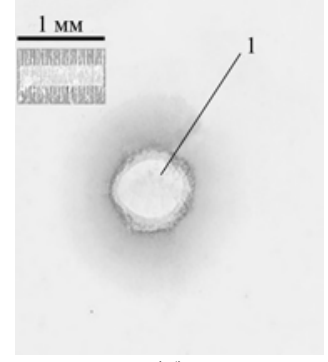

(б)

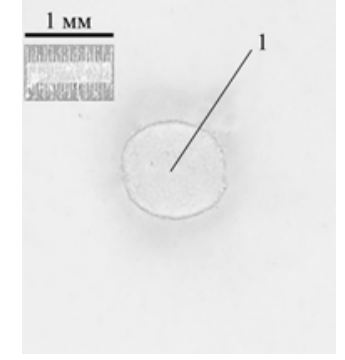

(в)

Рис. 3. Напыленный слой $S i$ на стеклянной пластинке $\left(E_{p}=1,5\right.$ Дж, $\tau_{p}=200$ мкс, $d_{p}=100$ мкм, $\left.\alpha=90^{\circ}\right)$ и разном расстоянии $S$ от стеклянной пластины до поверхности мишени, где: (а) $S=1$ мм; (б) $S=3$ мм; (в) $S=5$ мм; 1 - напыленный слой, поврежденный лазерным излучением (которое проходит стеклянную пластину насквозь и отражается от поверхности образца).

расстоянии $S=1-5$ мм (рис. 1) одновременно будут происходить:

- отражение лазерного излучения от поверхности мишени;

- нагрев и испарение поверхности мишени;

- оседание на поверхности стеклянной пластины плазменного облака и создание на ней тонкого слоя покрытия;

- испарение вновь созданного покрытия под действием лазерного излучения (которое проходит стеклянную пластину насквозь и отражается от поверхности образца), что делает данные условия обработки неприемлемыми для реализации технологии ИЛО (рис. 3).

Однако увеличение расстояния между мишенью и стеклянной пластиной (что ведет к одновременному увеличению размеров поперечного сечения лазерного луча, который проходит стеклянную пластину насквозь, и лазерного луча, который зеркально отражен от поверхности мишени) за счет уменьшения $W_{p}$ на eе поверхности позволяет наносить тонкие пленки на прозрачные (для данной длины волны лазерного излучения) материалы без их повреждения.
Кроме того, очевидно, что постепенное уменьшение угла $\alpha$ (рис. 1) ведет:

- к определенному уменьшению $W_{p}$ (за счет преобразования пятна фокусирования круглого сечения в эллипс и соответственно увеличения его площади);

- к изменению коэффициента отражения лазерного излучения;

- к отклонению оси отраженного от поверхности мишени лазерного луча и самого плазменного облака.

Последнее позволяет наносить напыленный слой на поверхность стеклянного образца без его повреждения. Так, на рис. 4 изображен напыленный слой Si на стеклянной пластинке, полученный при условии $E_{p}=1,5$ Дж, $\tau_{p}=200$ мкс, $d_{p}=100$ мкм и изменении $\alpha$ и $S$ (рис. 1).

Отметим, что сверхтонкая напыленная пленка кремния, которая появляется при осаждении плазменного облака на стеклянную пластину (находится на периферии видимого напыленного слоя) и которую не удается увидеть:

- при использовании микроскопа МБС-10 (рис. 3,4$)$ в отраженном и проходящем свете; 


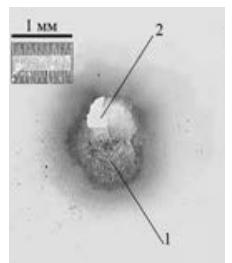

(a)

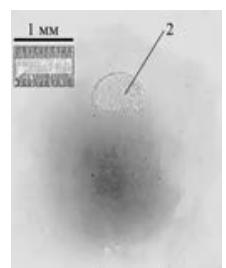

(б)

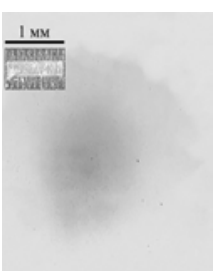

(B)

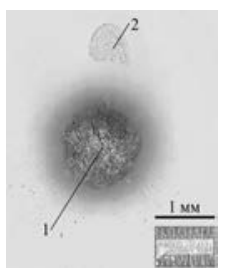

(г)

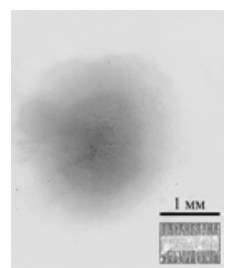

(д)

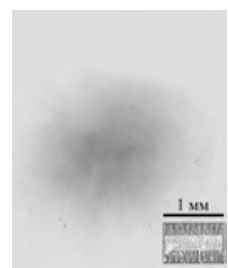

(e)

Рис. 4. Напыленный слой $\mathrm{Si}$ на стеклянной пластинке $\left(E_{p}=1,5\right.$ Дж, $\tau_{p}=200$ мкс, $d_{p}=100$ мкм) при разных углах наклона $\alpha$ и расстоянии $S$ : (а) $\alpha=60^{\circ}, S=1$ мм; (б) $\alpha=60^{\circ}, S=3$ мм; (в) $\alpha=60^{\circ}, S=5$ мм; (г) $\alpha=40^{\circ}, S=1$ мм; (д) $\alpha=40^{\circ}, S=3$ мм; (e) $\alpha=40^{\circ}, S=5$ мм; 1 - напыленный слой, поврежденный отраженным от поверхности мишени лазерным излучением; 2 - напыленный слой, поврежденный лазерным излучением, которое проходит пластину насквозь.

- при ее изучении с помощью микроскопа МИС-11 и интерферометра МИИ-4, изменяет оптические свойства стекла на границе поверхность-воздух. Наличие сверхтонкого слоя напыленного покрытия ведет к повреждению поверхности стеклянной пластины под действием лазерного излучения, которое фокусируется на поверхности мишени и проходит стеклянную пластину насквозь (позиция 2, рис. 4б,г). Возможно, толщина сверхтонкой пленки на периферии осажденного плазменного облака соответствует толщине пленки, которая образуется при классическом ИЛО материалов [1, 4-6]. Ее появление связано с уменьшением «плотности» плазмы, которое наблюдается при увеличении расстояния от:

- оси плазменного облака;

- зоны фокусирования лазерного излучения до поверхности заготовки, на которую напыляется материал мишени (при классическом ИЛО более 45 мм), и соответственно уменьшением количества атомов материала мишени, осевших на поверхности стеклянной заготовки (рис. 4).

Влияние созданной сверхтонкой напыленной пленки кремния на оптические свойства стекла К-8 подтверждается тем, что под действием лазерных импульсов $\left(E_{p}=1,5\right.$ Дж, $\tau_{p}=200$ мкс) и при фокусировании лазерного излучения на поверхности «чистой» пластины (пятно $d_{p}=500$ мкм) не наблюдается ее разрушение. Также ее повреждение не наблюдается и в случае облучения $\left(E_{p}=1,5\right.$ Дж, $\tau_{p}=200$ мкс) стеклянной пластины, расположенной на различных расстояниях от фокальной плоскости (расстояние $+/-2$ мм с шагом 0,5 мм).

Как и в предыдущем случае (рис. 1), увеличение $S$, ведущее к падению $W_{p}$ на поверхности стеклянной пластины, делает невозможным повреждение сверхтонкой напыленной пленки кремния (если она появляется) на поверхности заготовки (рис. 4в,д,е).

Известно [7], что стабильность результатов лазерной обработки материалов (как и любой технологии) целиком и полностью зависит от стабильности составляющих процесса.
Указанное справедливо и для ИЛО. Так, например, в случае обработки неподвижной мишени первый импульс сфокусированного лазерного излучения (в факторном пространстве, которое исследуется) формирует элементарное отверстие (рис. 5). В процессе испарения ось плазменного облака (пока не сформирован канал отверстия) перпендикулярна поверхности мишени (рис. 6а). Следующие импульсы сфокусированного лазерного излучения углубляют отверстие (рис. 5), ось которого совпадает с осью сфокусированного лазерного луча. Плазменное облако, которое образуется при испарении «дна» отверстия (и частично его стенок) «зажимается» поверхностью образовавшегося канала и направляется навстречу (вдоль оси) сфокусированному лазерному лучу (рис. 6б). Указанное (отклонение оси плазменного облака от начального положения) приводит к потере симметрии напыленного на подложку слоя материала мишени (рис. 7).

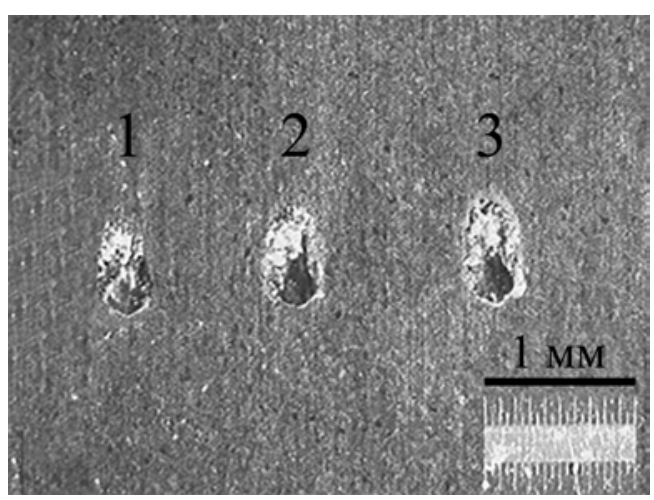

Рис. 5. Изображение отверстий, полученных в пластине $\mathrm{SiC}$, сфокусированным лазерным излучением $\left(E_{p}=1,0\right.$ Дж, $\tau_{p}=200$ мкс, $d_{p}=100$ мкм, $\alpha=40^{\circ}$ ) разным количеством импульсов, где $1,2,3$ - количество импульсов.

Именно поэтому при «многослойном» и многоимпульсном напылении мишень перемещалась в фокальной плоскости фокусирующего объектива от импульса к импульсу при $\alpha=40^{\circ}$, $S \geq 1$ мм.

Согласно методике исследований, толщина напыленного слоя определялась с помощью микроскопа МИС-11 и интерферометра МИИ-4. Так, например, на рис. 8 изображен центральный фрагмент профиля пленки кремния толщиной 


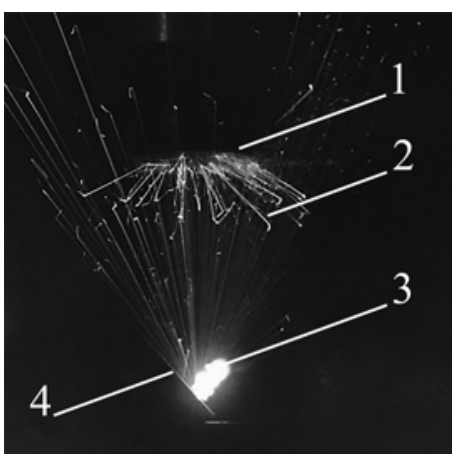

(a)

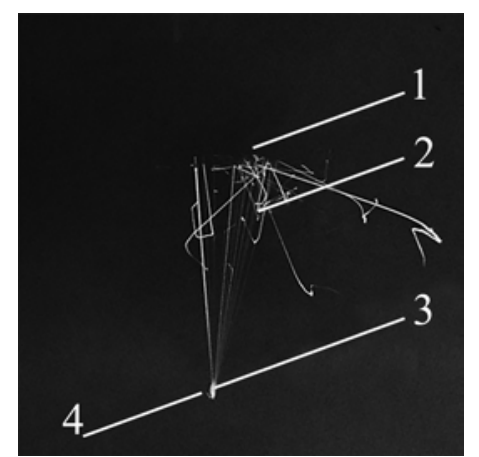

(б)

Рис. 6. Образование плазменного облака при многоимпульсной лазерной обработке $\left(E_{p}=1,0\right.$ Дж, $\tau_{p}=200$ мкс, $d_{p}=100$ мкм, $\alpha=40^{\circ}$ ) образцов из $\mathrm{SiC}$ первым (а) и третьим (б) импульсами, где 1 - фокусирующий объектив; 2 - «брызги» материала; 3 - плазменное облако; 4 - образец.

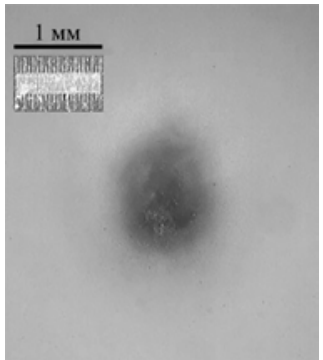

(a)

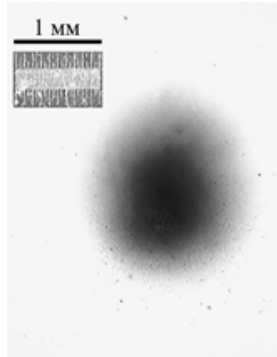

(б)

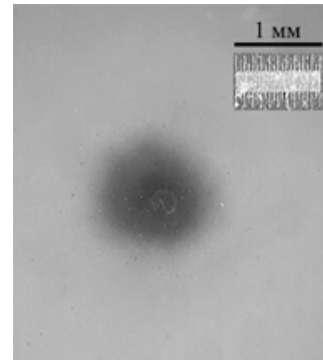

(в)

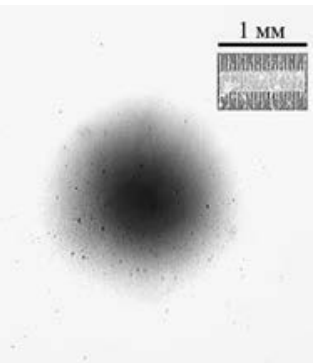

(г)

Рис. 7. Изображение напыленного слоя Si на стеклянной пластине в отраженном (а, в) и проходящем (б, г) свете, для трех лазерных импульсов $\left(E_{p}=1,5\right.$ Дж, $\tau_{p}=200$ мкс, $d_{p}=100$ мкм, $\alpha=40^{\circ}, S=2$ мм), сфокусированных на неподвижную (а, б) и подвижную (в, г) мишени.

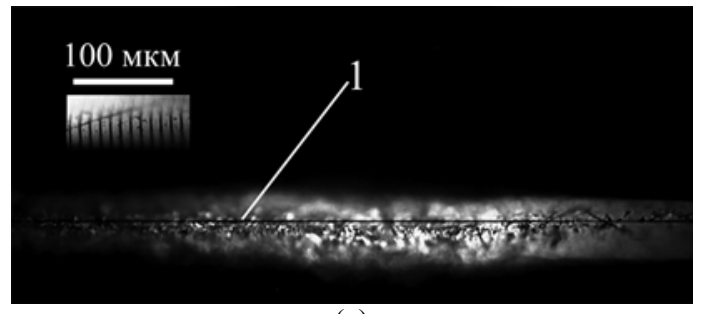

(a)

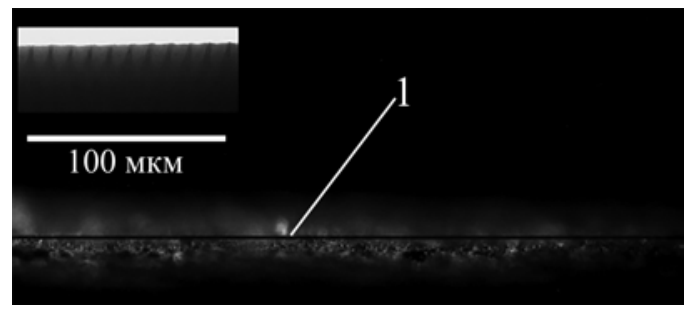

(б)

Рис. 8. Изображения центрального фрагмента профиля пленки $\mathrm{Si}$ на стеклянной пластине $\left(E_{p}=1,5\right.$ Дж, $\tau_{p}=200$ мкс, $d_{p}=100$ мкм, $\alpha=40^{\circ}, 1$ импульс, $S=2$ мм), полученные с помощью различных объективов микроскопа МИС-11, где: (a) - изображение, полученное с помощью объектива с фокусным расстоянием 8,16 мм; (б) - изображение, полученное с помощью объектива с фокусным расстоянием 4,25 мм; 1 - линия поверхности стекла.

8 мкм, а на рис. 9 - зависимость максимальной высоты напыленного слоя от количества лазерных импульсов (или количества осажденных плазменных облаков) для разного расстояния $S$ от стеклянной пластины до поверхности мишени. Как отмечалось ранее, при расстоянии до мишени $S=1$ мм и $\alpha=40^{\circ}$ в центре осажденного плазменного облака (рис. 4г) за счет отраженного от зоны обработки лазерного излучения происходит повреждение нанесенного покрытия. Указанное повреждение исчезает при испарении мишени следующими лазерными импульсами (рис. 10). Данное явление можно объяснить рядом причин:

- уменьшением количества лазерной энергии, которая достигает поверхности мишени, что, вероятно, происходит за счет роста размеров поврежденного напыленного слоя, через который проходит лазерное излучение (рис. 4г, 10), и соответственно роста его потерь;
- изменением размера пятна фокусирования лазерного излучения, которое происходит из-за трансформации рельефа поврежденного участка, ведущего к изменению формы каустики;

- созданием (предыдущими импульсами) переходного слоя между аморфным $\mathrm{SiO}_{2}$ (материалом подложки) и кристаллическим $\mathrm{Si}$ (который остался при испарении осажденного плазменного облака).

Известно [8], что яркость отдельной точки фотографического изображения зависит от свойств трехмерного оригинала, условий его освещения, коэффициентов отражения (диффузионного и зеркального) точек поверхности объекта, их пространственных координат. В нашем случае при равномерном освещении поверхности заготовки рассеянным светом (на которую нанесен слой одного материала и соответственно с одним коэффициентом отражения) мы можем сопоставить измеренную 


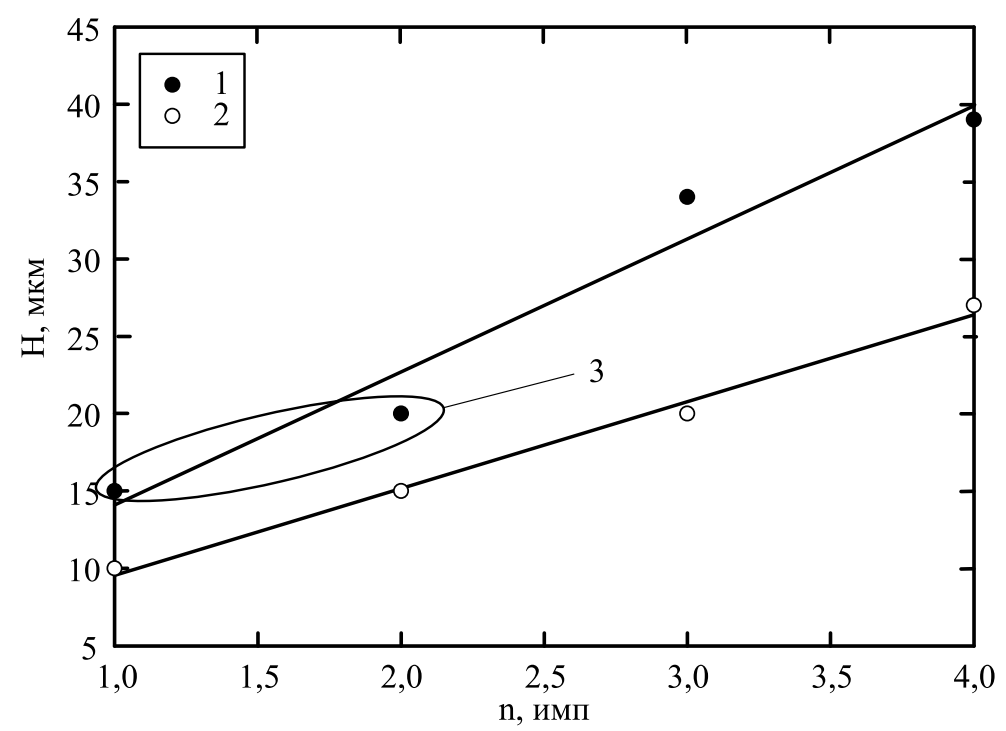

Рис. 9. Зависимость максимальной высоты пленки кремния $H$ от количества импульсов $n\left(E_{p}=1,5\right.$ Дж, $\tau_{p}=200$ мкс, $d_{p}=100$ мкм, $\alpha=40^{\circ}$ для разного расстояния $S$, где $1-S=1$ мм; $2-S=2$ мм; 3 - зона поврежденного покрытия.

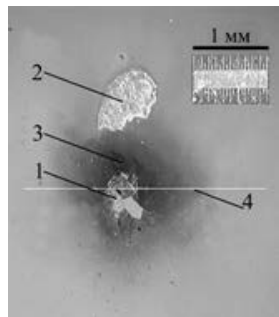

(a)

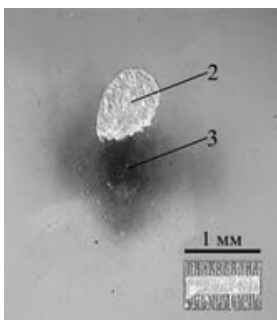

(б)

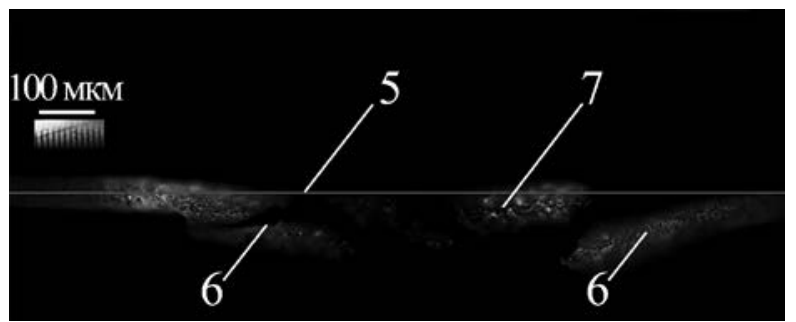

(в)

Рис. 10. Изображение напыленного слоя Si на стеклянной пластине при условиях $\left(E_{p}=1,5\right.$ Дж, $\tau_{p}=200$ мкс, $d_{p}=100$ мкм, $\alpha=40^{\circ}, S=1$ мм) и подаче двух (а) и трех (б) импульсов на мишень, а также профиль поврежденного участка (в), где 1 - напыленный слой, поврежденный отраженным от поверхности мишени лазерным излучением; 2 - напыленный слой, поврежденный лазерным излучением, которое проходит пластину насквозь; 3 - напыленный слой; 4 - линия определения профиля поврежденного участка; 5 - поверхность стекла; 6 - «отделенная» от стекла часть покрытия; 7 - остатки пленки Si.

высоту нанесенного слоя вдоль линии измерений (рис. 8-10) с яркостью его изображения (рис. 3, 4, 10). В дальнейшем, экстраполируя полученные данные на всю поверхность напыленного слоя, получим трехмерное изображение плоской картины напыленного слоя (рис. 3, 4, 10), то есть зависимость его высоты от пространственных координат $H(x, y)$.

Полученные изображения нанесенных пленок обрабатывались с помощью специальной программы, которая:

- читала файл, содержащий изображение;

- масштабировала его по пространственным координатам;

- в соответствии с проведенными измерениями (рис. 8-10) преобразовывала яркость отдельной точки в высоту напыленного слоя и представляла результат в виде ${ }^{*}$.ТХT файла (для дальнейшей обработки) или в виде трехмерных графиков. Так, на рис. 11 изображены напыленные пленки $\mathrm{Si}$ на стеклянной пластинке $\left(E_{p}=1,5\right.$ Дж, $\tau_{p}=200$ мкс, $d_{p}=100$ мкм, $\alpha=40^{\circ}$, $S=2$ мм) и их преобразование в трехмерный вид $H(x, y)$.

Таким образом, зная $H(x, y)$, в зависимости от режимов обработки мы можем создавать осажденное покрытие больших размеров с «равномерной» толщиной путем осаждения плазменных облаков на перемещающуюся подложку по определенному закону. Например, за счет перемещения подложки с определенным шагом, причем в случае:

- «одноимпульсной» обработки (то есть в одно место осаждается одно плазменное облако) и желательная толщина напыленного слоя достигается за счет необходимого количества проходов;

- «многоимпульсной» обработки, то есть в одно место осаждается несколько плазменных облаков, количество которых (рис. 9) и определяет желаемую толщину покрытия, а также их комбинации.

Для определения оптимальных режимов обработки использовали зависимости $H(x, y)$, которые были получены для различных режимов облучения. Причем, согласно методике проведения исследований:

- использовали усредненные зависимости высоты напыленного слоя от пространственных координат $H_{\text {middle }}(x, y)$, которые были получены при обработке трех изображений напыленных пленок; 


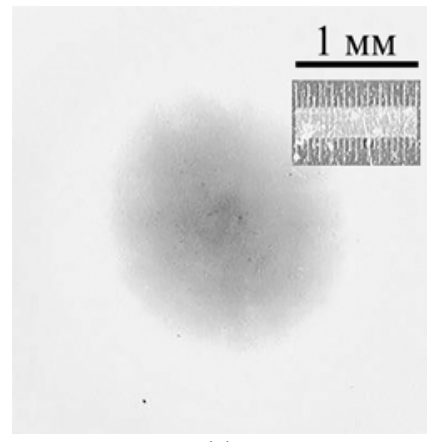

(a)

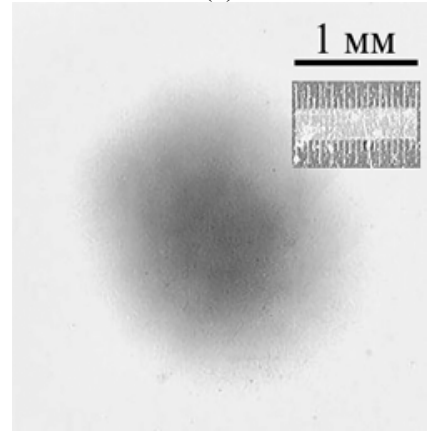

(в)
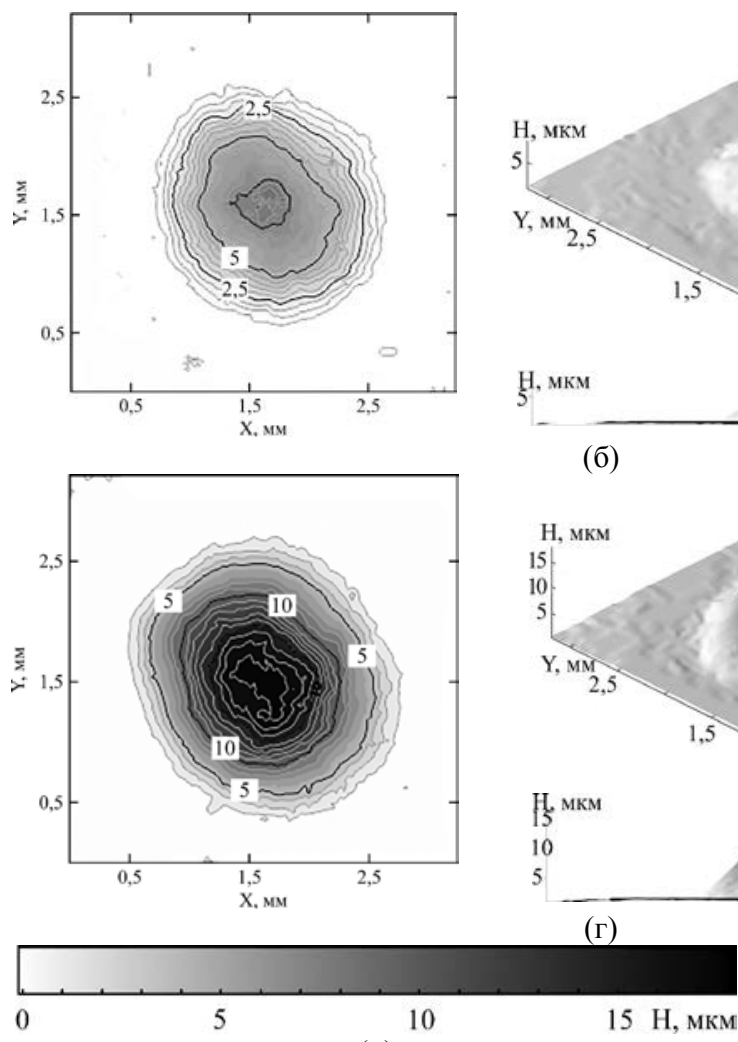

(д)

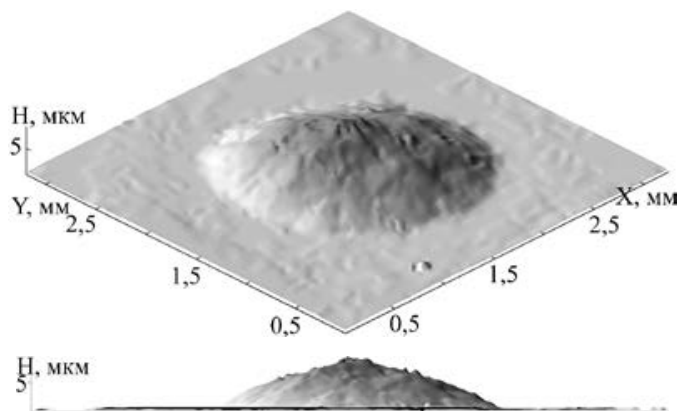

(б)

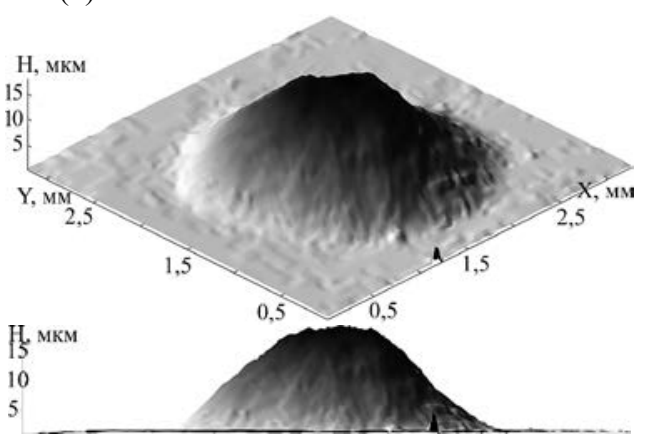

$(\Gamma)$

15 Н, мкм

Рис. 11. Напыленные пленки Si на стеклянной пластине $\left(E_{p}=1,5\right.$ Дж, $\tau_{p}=200$ мкс, $d_{p}=100$ мкм, $\alpha=40^{\circ}, S=2$ мм) и их трехмерный вид $H(x, y)$, где: (а) - пленка, полученная за 1 импульс и (б) - ее трехмерное изображение; (в) - пленка, напыленная за 3 импульса и (г) - ее трехмерное изображение; (д) - масштаб по высоте.

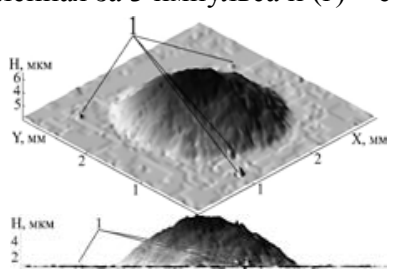

(a)

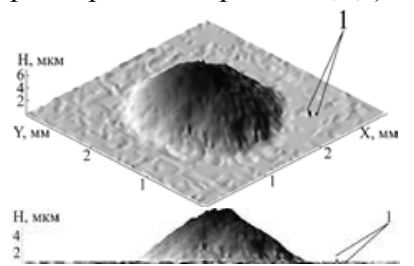

(б)

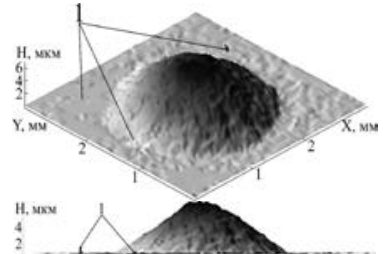

(в)

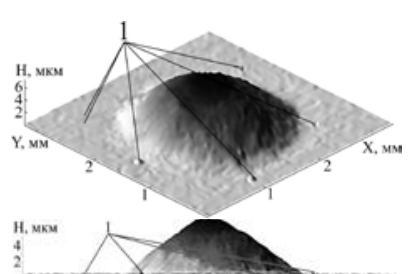

$(\Gamma)$

(д)

Рис. 12. Трехмерный вид напыленной пленки Si на стеклянной пластине $\left(E_{p}=1,5\right.$ Дж, $\tau_{p}=200$ мкс, $d_{p}=100$ мкм, $\alpha=40^{\circ}$, $S=2$ мм), где 1 - осажденные частицы материала мишени; (а), (б), (в) - пленка, полученная за 1 импульс $H_{i}(x, y)$; (г) - поверхность, которая описывает средние значения высот $H_{\text {middle }}(x, y)$ точек пленки; (д) - масштаб по высоте.

- считали, что при наложении последующего слоя покрытия на уже существующий суммарная высота изменялась с учетом углового коэффициента зависимостей 1,2 , которые приведены на рис. 9.

Так, на рис. 12 приведены зависимости высоты напыленного слоя Si от пространственных координат $H(x, y)$, полученные за один импульс, и их усредненная зависимость $H_{\text {middle }}(x, y)$, а на рис. 13 - рассчитанные трехмерные изображения пленок Si на стеклянной пластинке, полученные при использовании зависимости $H_{\text {middle }}(x, y)$ (рис. 12г), которая накладывается на пластину с шагом (рис. 14) Step $_{O X}=$ Step $_{O Y}=0,5 \mathrm{mм}($ DStep $=0,0 \mathrm{mм}), \mathrm{c}$ простым суммированием зависимостей $H(x, y)$ и с учетом углового коэффициента зависимости 2 , приведенной на рис. 9.
«Идеальное» распределение $H(x, y)$ (рис. 11, 12), которое формируется ядром плазменного облака (позиция 3, рис. 6), искажается застывшими одиночными элементами мишени. Появление данных элементов носит случайный характер, а сами элементы представляют собой застывшие «брызги» жидкой фазы материала мишени, которые вылетели из зоны испарения (позиция 2, рис. 6). В нашем случае $\left(E_{p}=1,5\right.$ Дж, $\tau_{p}=200$ мкс, $d_{p}=100$ мкм) при нагреве и последующем испарении $\mathrm{Si}$ образуется значительное количество жидкой фазы, которая вытесняется и «выбрасывается〉 парами плазмы из зоны нагрева в открытую атмосферу. Отметим, что при реализации ИЛО минимизация количества жидкой фазы, возникающей в зоне лазерного нагрева, достигается за счет использования лазеров, 


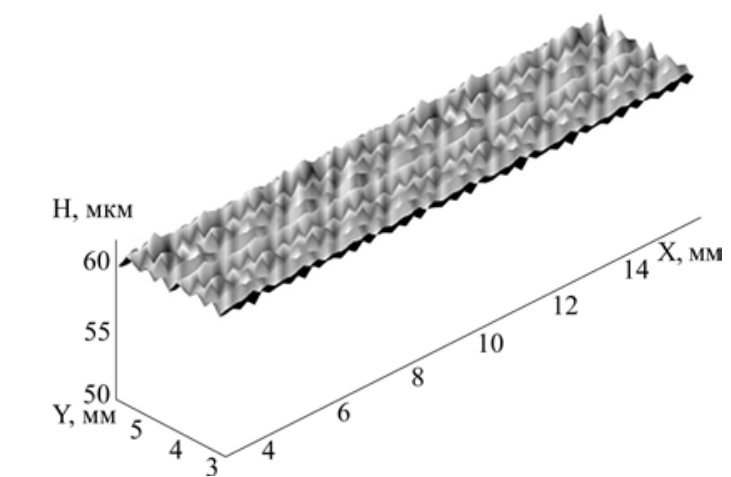

(a)

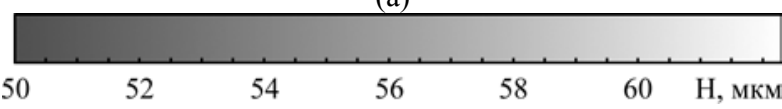

(B)

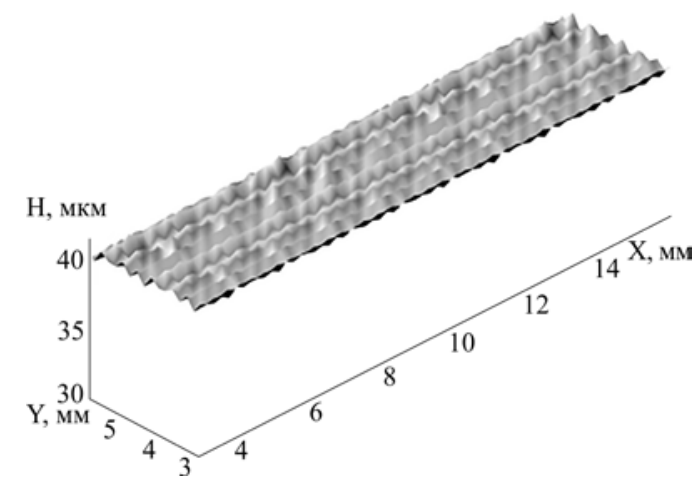

(б)

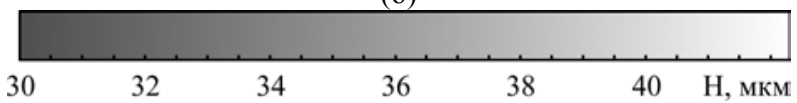

(г)

Рис. 13. Расчетный трехмерный вид напыленной пленки $\mathrm{Si}$, полученной с использованием $H_{\text {middle }}(x, y)\left(E_{p}=1,5\right.$ Дж, $\tau_{p}=200$ мкс, $d_{p}=100$ мкм, $\alpha=40^{\circ}, S=2$ мм, Step $O X=$ Step $_{O Y}=0,5$ мм, DStep $=0,0$ мм), где (а) - простое суммирование зависимостей; (б) - с использованием учета наклона зависимостей $H(n)$; (в) и (г) - масштабы по высоте.

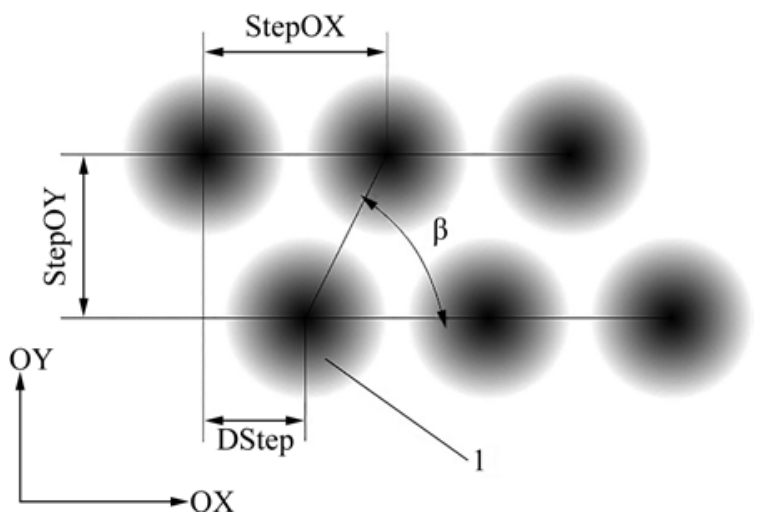

Рис. 14. Независимые переменные при поиске оптимальных режимов обработки, где 1 - напыленный слой.

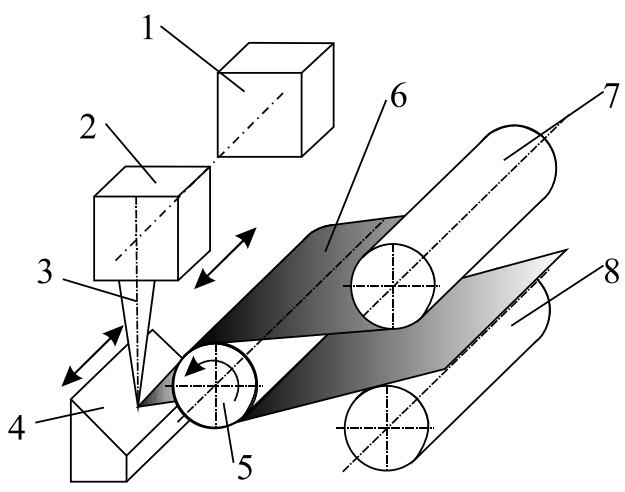

(a)

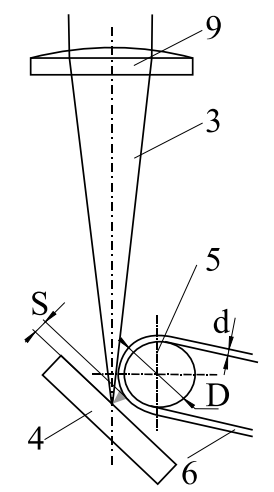

(б)

Рис. 15. Схема способа нанесения покрытия на тонкие пленки методом ИЛО, где 1 - лазер; 2 - подвижный оптический блок; 3 - сфокусированное лазерное излучение; 4 - подвижная мишень; 5 - рабочий вал диаметром $D$; 6 - пленка толщиной $d ; 7,8$ - дополнительные валики; 9 - линза.

которые генерируют импульсы наносекундной длительности [1, 4-6]. А полное отсутствие жидкой фазы в зоне лазерного нагрева - при использовании лазеров, которые генерируют сверхкороткие импульсы (пико- и фемтосекундной длительности) [6].

Отметим, что аппроксимация полученных значений $H_{\text {middle }}(x, y)$ экспоненциальной поверхностью $H_{\text {calc }}(x, y)(1)$ позволяет с высокой степенью точности описывать форму напыленного участка.

$$
H_{\text {calc }(x, y)}=A \times \exp \left(-B \times\left(\left(\frac{x-x_{0}}{r_{x}}\right)^{2}+\left(\frac{y-y_{0}}{r_{y}}\right)^{2}\right)\right)
$$

где $A, B, r_{x}, r_{y}$ - коэффициенты уравнения; $x_{0}, y_{0}-$ координаты центра поверхности.

Так, например, поверхность $H_{\text {middle }}(x, y)$, изображенная на рис. 12г, аппроксимируется зависимостью $H_{\text {calc }}(x, y)$ с коэффициентами $A=7,52$ мкм, $B=1,68 \mathrm{мM}, r_{x}=1,04 \mathrm{мм}$, $r_{y}=1,07 \mathrm{Mм}, x_{0}=1,64 \mathrm{Mм}, y_{0}=1,57$ мм. При этом средняя ошибка аппроксимации (MPE) не 


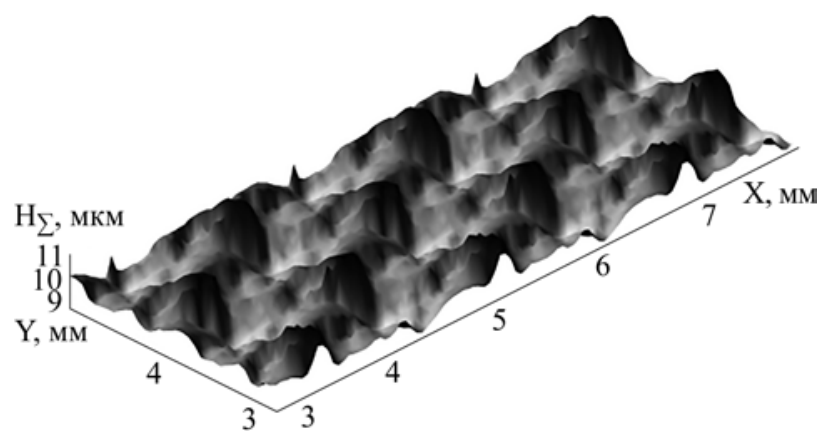

(a)

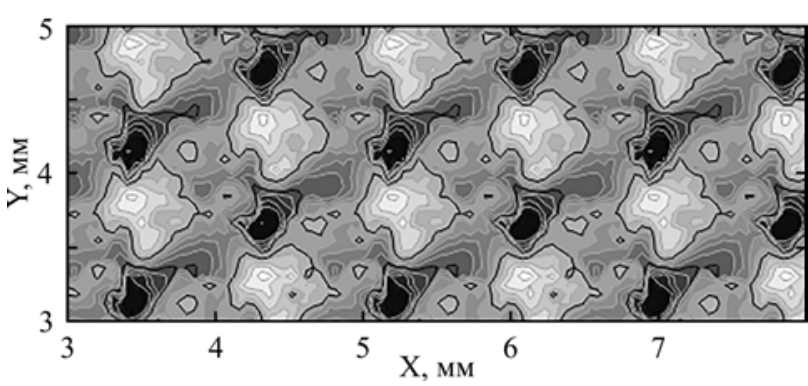

(б)

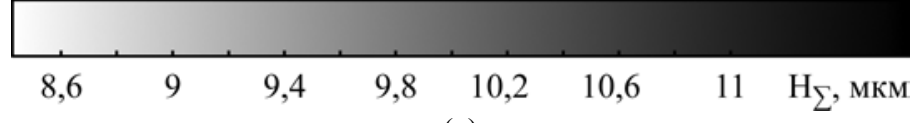

(B)

Рис. 16. Расчетный трехмерный вид напыленной пленки $\mathrm{Si}$ средней толщиной 10 мкм $\left(E_{p}=1,5\right.$ Дж, $\tau_{p}=200$ мкс, $d_{p}=100$ мкм, $\alpha=40^{\circ}, S=2$ мм, Step ${ }_{O X}=1,8$ мм, Step $O Y=0,5$ мм, DStep $=0,9$ мм), где (a) - трехмерный вид поверхности; (б) - карта распределения равных уровней; (в) - масштаб по высоте.

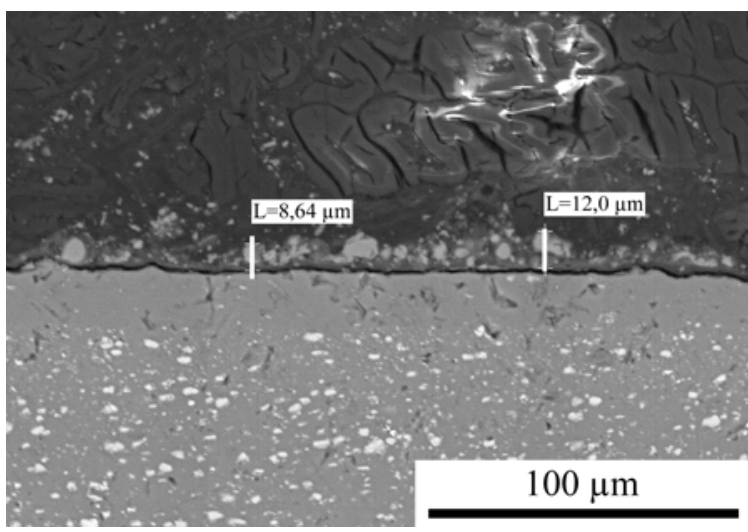

Рис. 17. Микроструктура поперечного сечения пленки $S i$ на алюминиевой подложке $\left(E_{p}=1,5\right.$ Дж, $\tau_{p}=200$ мкс, $d_{p}=100$ мкм, $\alpha=40^{\circ}, S=2$ мм, Step $O X=1,8$ мм, Step $O Y=0,5$ мм, DStep = 0,9 мм). Толщина подложки -1 мм.

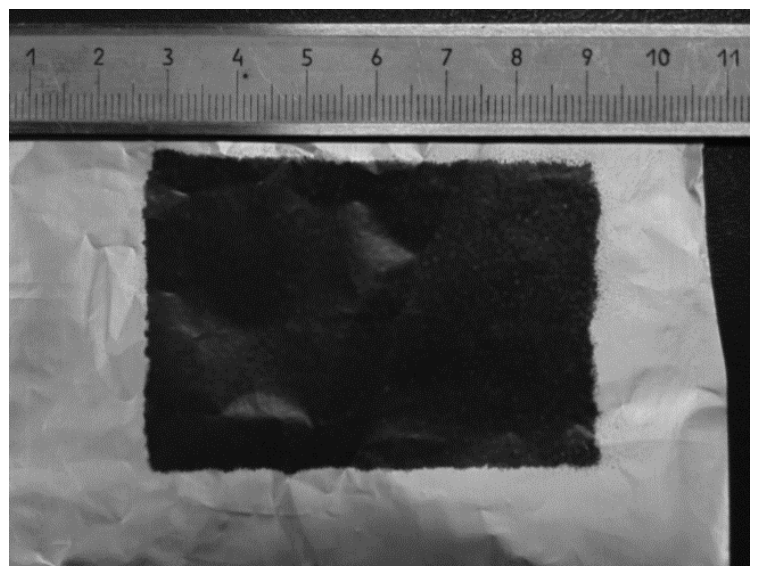

Рис. 18. Внешний вид напыленной пленки Si (размер $40 \times 60$ мм) средней толщиной 9,75 мкм на алюминиевой фольге толщиной 10 мкм $\left(E_{p}=1,5\right.$ Дж, $\tau_{p}=200$ мкс, $d_{p}=100$ мкм, $\alpha=40^{\circ}, S=2$ мм, Step $O X=1,8$ мм, Step $O Y=0,5$ мм, DStep $=0,9$ мм).

превышает 7,8\%, а средняя абсолютная ошибка аппроксимации (МАРЕ) не превышает $12 \%$, что свидетельствует о приемлемости аппроксимации экспоненциальной поверхностью $H_{\text {calc }}(x, y)$ полученных зависимостей $H(x, y)$ [9]. Отметим, что:

- аппроксимация $H(x, y)$ квадратичной поверхностью ведет к более чем трехкратному (по абсолютной величине) увеличению МРЕ (до $27 \%$ ) и более чем четырехкратному увели- чению МАРЕ (до 58\%), что свидетельствует о невозможности описания поверхностью второго порядка «мягкого» перехода поверхности заготовки в напыленный слой;

- описание $H(x, y)$ простой зависимостью (1), несмотря на ее низкие MPE и MAPE, не позволяет учитывать застывшие одиночные элементы мишени.

Поэтому для более точного описания поверхностей при расчетах использовали зависимости 
$H(x, y)$ в виде трехмерных массивов, которые содержали координаты точек. Для наших условий обработки $\left(E_{p}=1,5\right.$ Дж, $\tau_{p}=200$ мкс, $d_{p}=100$ мкм, $\alpha=90^{\circ}-40^{\circ}, S=1-4$ мм) и материала мишени (Si) максимальная высота осажденных одиночных частиц не превышает 0,5-2 мкм (рис. 3, 4, 7, 10-12), что значительно меньше высоты ядра покрытия. Благодаря этому (случайное появление и малая высота) наличие застывших элементов мишени на напыленном участке не оказывает значительного влияния на высоту покрытия при нанесении его на большую площадь.

При простом суммировании напыленных одиночных пятен (рис. 12б), благодаря малому шагу их перемещения (Step OX $=$ Step $_{O Y}=0,5$ мм) по поверхности заготовки (приблизительно в 2 раза меньше радиуса $r_{x},\left(r_{y}\right)$ одиночного пятна напыления), суммарная высота напыленного слоя составит более 60 мкм (рис. 13a). При учете угла наклона зависимости $H(n)$ (для данных условий обработки) высота напыленного слоя составит около 42 мкм (рис. 13б). Несмотря на разную высоту напыленных слоев (рис. 13), внешний вид образованных напыленных поверхностей будет подобным.

В качестве целевой функции использовали минимум отклонения $H_{S Q}(2)$ желаемой толщины напыленного слоя $Z=H$ от высоты рассчитанной поверхности $H_{\Sigma}(x, y)$, созданной многими пятнами напыления. В качестве независимых

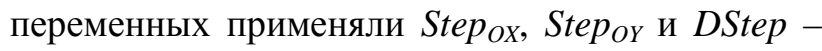
шаг между отдельными напыленными пятнами и сдвиг между ними (рис. 14). Величины Step Step $_{O Y}$ и DStep определяют угол наклона пятен напыления $\beta$ вдоль оси $O X$. Отметим, что при нанесении покрытия большой площади на тонкие пленки (рис. 15) [2, 3], учитывая особенности реализации данной технологии, шаг перемещения отдельных пятен Step соответствует перемещению оптического блока 2 вместе с фокусирующей линзой 9 (рис. 15) вдоль оси цилиндра 5 (рис. 15), а шаг перемещения заготовки Step $о$ - вращению цилиндра.

$$
H_{S Q}=\min \left(H-H_{\Sigma}(x, y)\right)^{2} .
$$

Очевидно, что для решения задачи (2) наиболее целесообразно использовать методы нелинейного программирования. Для поиска оптимальных значений независимых переменных использовали метод Хука-Дживса [10], который не требует аналитических выражений производных функции цели, а для учета ограничений на интервалы изменения независимых переменных (считали, что лежат в интервале от 0,5 до 1,5 мм) применяли метод «штрафных» функций [10].

Благодаря расчетам установлено, что минимальное колебание высоты напыленного слоя $H_{S}(x, y)$ наблюдается при условии, когда $S_{t e p}$ и и Step $O Y$ не превышают радиус напыленного одиночного пятна (рис. 13). Как отмечалось paнее, в этом случае из-за наложения напыленных пятен растет суммарная высота $H_{S}$ напыленного слоя. Очевидно, что в случае, когда происходит поиск Step OXX $_{\text {Step }}$ и и DStep, при формировании слоя заданной высоты (для данных $E_{p}, \tau_{p}, d_{p}, \mathrm{~F}, \alpha, S$ и при одно- и трехимпульсном напылении - рис. 9) величина независимых переменных будет расти. Так, например, для получения напыленного слоя высотой $H_{\Sigma}=10$ мкм (при условии $E_{p}=1,5$ Дж, $\tau_{p}=200$ мкс, $d_{p}=100$ мкм, $\alpha=40^{\circ}, S=2$ мм) с минимальным колебанием высоты оптимальные значения независимых переменных равны: Step $_{O X}=1,8 \mathrm{мm}$, Step $_{O Y}=0,5$ мм и DStep $=0,9$ мм. При этом угол наклона напыленных пятен $\beta=30^{\circ}$.

Экспериментальная проверка показала, что на данных режимах обработки $\left(E_{p}=1,5\right.$ Дж, $\tau_{p}=200$ мкс, $d_{p}=100$ мкм, $\alpha=40^{\circ}, S=2$ мм, Step $_{O X}=1,8$ мм, Step $O$ OY $=0,5$ мм и DStep $=0,9$ мм) минимальная высота напыленного слоя составляет 8,42 мкм, максимальная - 11,63 мкм, среднее значение - 9,75 мкм. Так, для данных режимов обработки на рис. 16 приведено рассчитанное распределение толщины напыленного слоя Si от пространственных координат, на рис. 17 - микроструктура поперечного сечения пленки Si на алюминиевой подложке толщиной 1 мм, на рис. 18 - внешний вид напыленной пленки кремния размерами 40×60 мм на алюминиевой подложке $\delta=0,01$ мм.

\section{ВЫВОДЫ}

В результате проведенных исследований установлено, что в пространстве проектирования для технологии ИЛО:

- появляется возможность нанесения слоев кремния различной толщины на гибкую тонкую заготовку «неограниченных» размеров;

- разработан метод определения режимов нанесения покрытия, который обеспечивает минимальное колебание толщины напыленного слоя;

- для одинаковых условий обработки (расстояние между мишенью и заготовкой, энергия импульса и пр.) наблюдаются минимальное колебание толщины напыленного слоя и рост его высоты при уменьшении расстояния между «одиночными» напыленными пятнами; 
- распределение высоты слоя осажденного материала мишени на поверхности заготовки (в «одиночном» напыленном пятне) в пространственных координатах можно аппроксимировать экспоненциальной поверхностью;

- на поверхности заготовки, на периферии напыленного пятна, создается сверхтонкий слой из элементов материала мишени, толщину которого невозможно определить с помощью оптических методов.

\section{КОНФЛИКТ ИНТЕРЕСОВ}

Авторы заявляют, что у них нет конфликта интересов.

\section{ЛИТЕРАТУРА}

1. LIA Handbook on Laser Materials Processing, Ready J.F. and Farson D.F., eds. LIA Magnolia Publishing, Inc., 2001, 715 p.

2. Zhuk, R., Zhang, Q., Anyakin, M., Yao, J., Laser deposition of thin Al-Si photo-voltaic coatings, Book of $40^{\text {th }}$ International MATADOR Conference on Advanced Manufacturing, July 8-10, 2019, Handzhou, China, p. 188.

3. Скляр, М.О., Байбакова, Е.В., Жук, Р.О., Анякин, Н.И., и др., Способ нанесения покрытия лазерным излучением. Патент України №114446 (Бюл. № 5/2017 10.03.2017, заявка: u201609201 02.09.2016).

4. Greer, J.A., History and current status of commercial pulsed laser deposition equipment, J. Phys. D Appl. Phys., 2014, vol. 47, 034005. doi:10.1088/00223727/47/3/034005.

5. Hofmann-Urlaub, S., Zhang, Y., Wang, Zh., Kressdorf, B., et al., Fabrication of tin-based halide perovskites by pulsed laser deposition, Appl. Phys. A, 2020, vol. 126, no. 7, p. 553 https://doi.org/10.1007/s00339-020-03699-9
6. Benett, D., Nouar, R., Nechache, R., Pepin, H., et al., Combined magnetron sputtering and pulsed laser deposition of $\mathrm{TiO}_{2}$ and BFCO thin films, Sci. Rep., 2017, vol. 7, 2503. doi:10.1038/s41598-017-02284-0.

7. Грановский, В.А., Сирая, Т.Л., Методы обработки экспериментальных данных. JI.: Энергоатомиздат, 1990.

8. Иванов, В.П., Батраков, А.С., Полищук, Г.М., Трехмерная компьютерная графика. М.: Радио и связь, 1995, 224 с.

9. Swamidass, P.M., Encyclopedia of Production and Manufacturing Management, Boston: Springer, 2000. https://doi.org/10.1007/1-4020-0612-8_580.

10. Химмельблау, Д., Прикладное нелинейное программирование. М.: Мир, 1975, 534 с.

\section{Summary}

The present paper deals with the selection of laser beam processing parameters that guarantee the formation of thin coatings with even thickness by means of the laser beam deposition. Laser deposition was carried out in the atmosphere of $\mathrm{Ar}$ and $\mathrm{Si}$ and $\mathrm{SiC}$ samples were used as a source for the deposited material. Preliminary experiments show that there is a direct dependency between the brightness of the image of the deposited layer (at uniform illumination conditions) and its thickness. Therefore, it was possible to determine the thickness of the deposited layer for various processing parameters and to calculate the laws of motion of the work piece that guarantee the deposition of the layer with a minimal deviation of thickness from the required value. Calculated parameters were successfully used for the deposition of coatings on a substrate of a relatively large area.

Keywords: laser, laser technology, pulsed laser deposition, optimization of technological processes 